J. Korean Math. Soc. 48 (2011), No. 1, pp. 23-31

DOI 10.4134/JKMS.2011.48.1.023

\title{
ON RIEMANNIAN MANIFOLDS OF \\ CONSTANT NEGATIVE CURVATURE
}

\author{
Reza Mirzaie
}

ABSTRACT. In this paper, we study the fundamental group and orbits of cohomogeneity two Riemannian manifolds of constant negative curvature.

\section{Introduction}

Let $M^{n}$ be a complete and connected $n$-dimensional Riemannian manifold and $G \subset \operatorname{Iso}(M)$ be a closed and connected Lie subgroup of Iso $(M)$ (the group of isometries of $M$ ). We say that $M$ is a $G$-manifold of $G$-cohomogeneity $m$, if the maximum of the dimensions of orbits $G(x), x \in M$ is $n-m$. When $m=0$ or 1 and $M$ is negatively curved, there are interesting relations between topological properties of $M$ and orbits of the action of $G$ on $M$. If $m=0$, then $M$ is a homogeneous $G$-manifold. S. Kobayashi proved in [8] that $a$ homogeneous Riemannian $G$-manifold $M$ of negative curvature is diffeomorphic to $\mathbb{R}^{n}, n=\operatorname{dim} M$. The authors of [14] got many results about cohomogeneity one Riemannian $G$-manifolds of negative curvature. Among other results, they proved that if $M^{n}, n \geq 3$ is a complete and connected non-simply connected cohomogeneity one Riemannian manifold of negative curvature, then either $M$ is diffeomorphic to $\mathbb{R}^{k} \times T^{r}, r+k=n$, or $\pi_{1}(M)=\mathbb{Z}$ and the principal orbits are covered by $S^{n-2} \times \mathbb{R}$. There are little conclusions about topological properties of cohomogeneity two Riemannian manifolds of negative curvature. In this paper we are interested in cohomogeneity two Riemannian manifolds of constant negative curvature. Our main result is Theorem 3.6.

\section{Preliminaries}

In this paper, $M$ is a complete and connected Riemannian manifold. Let $G$ be a connected and closed Lie subgroup of isometries of $M$ and $\widetilde{M}$ be the universal Riemannian covering manifold of $M$, by the covering map $\pi: \widetilde{M} \rightarrow$ $M$. We will denote the deck transformation group of this covering by $\Delta$. It is

Received December 20, 2008.

2010 Mathematics Subject Classification. 53C30, 57S25.

Key words and phrases. Riemannian manifold, Lie group, sectional curvature. 
well known that $\Delta$ is isomorphic to the fundamental group $\pi_{1}(M)$ of $M$. We now mention some facts that we will use in the sequel.

Fact 2.1. Let $G$ be a connected Lie group which acts by isometries on $M$. Then

(a) There exists a connected covering group $G^{\prime}$ of $G$, such that $G^{\prime}$ acts by isometries on $\widetilde{M}$. If $g^{\prime} \in G^{\prime}$, then $g^{\prime}$ commutes with each deck transformation $\delta \in \Delta$.

(b) If the action of $G$ on $M$ is of cohomogeneity $m$, then the action of $G^{\prime}$ on $\widetilde{M}$ is of cohomogeneity $m$. For each orbit $\widetilde{D}$ in $\widetilde{M}, \pi(\widetilde{D})$ is an orbit in $M$, and each orbit in $M$ is the image of an orbit of $\widetilde{M}$ under the map $\pi$.

(c) If $G$ has a fixed point in $M$, then $G^{\prime}=G$ and Fix $\left(G^{\prime}, \widetilde{M}\right)$ is the full inverse image of Fix $(G, M)$ (where Fix $(G, M)=\{x \in M: G(x)=x\})$.

(d) Following Fact(c), if $G^{\prime}$ has only one fixed point in $\widetilde{M}$, then $\widetilde{M}=M$.

Proof. The group $G^{\prime}$ is defined as the compact case in [3] page 63. The proof of (a) and of (c) can be made in the same way as the proof of Theorem 9.1 in [3]. (b) is a simple consequence of the definition of $G^{\prime}$. For the proof of (d), note that if $x_{0}$ is the fixed point of $G$ in $M$, then by (c), $\pi^{-1}\left(x_{0}\right)$ must be a one point set. Thus $\pi$ is one to one and $\widetilde{M}=M$.

The following theorem is about cohomogeneity-one Riemannian manifolds. For the definition of singular and principal orbits and details about cohomogeneity-one Riemannian manifolds, we refer to [2], [12] or [14].

Theorem 2.2 ([12]). Let $\mathbb{R}^{n}$ be of cohomogeneity one, under the action of a connected and closed Lie group $G$ of isometries. Then, either each principal orbit is isometric to $\mathbb{R}^{n-1}$ and there are no singular orbits, or each principal orbit is isometric to $S^{k}(c) \times \mathbb{R}^{n-k-1}$ ( $k$ is invariant for all orbits, $c>0$ depends on orbits) and there is a unique singular orbit isometric to $\mathbb{R}^{n-k-1}$.

Fact 2.3. Let $M$ be a negatively curved Riemannian manifold and $\widetilde{M}$ be its universal Riemannian covering manifold. If all of elements of a non trivial subgroup $H$ of $\pi_{1}(M)(=\Delta)$ leaves invariant a fixed geodesic $\gamma$, then $H$ is infinite cyclic.

Proof. See [5], page 261, Lemma 3.5.

Theorem 2.4 ([8]). If $M^{n}$ is a homogeneous Riemannian manifold of negative curvature, then it is diffeomorphic to $\mathbb{R}^{n}$.

Theorem 2.5 ([17]). If $M^{n}$ is a homogeneous Riemannian manifold of nonpositive curvature, then it is diffeomorphic to $\mathbb{R}^{n} \times T^{n-k}, k \geq 0$.

Consider the Lorentzian space $\mathbb{R}^{n, 1}\left(=\mathbb{R}^{n+1}\right)$ with a non-degenerate scalar product $\langle$,$\rangle defined by:$

$$
\langle x, y\rangle=-x_{1} y_{1}+\sum_{i=2}^{n+1} x_{i} y_{i}
$$


It is well known that any simply connected Riemannian manifold of constant negative curvature, $c<0$, is isometric to the hyperbolic space of curvature $c$ defined by:

$$
H^{n}(c)=\left\{x \in \mathbb{R}^{n, 1}:\langle x, x\rangle=-r^{2}\right\}, c=-\frac{1}{r^{2}} .
$$

We recall that infinity $\widetilde{M}(\infty)$ of a simply connected negatively curved Riemannian manifold $\widetilde{M}$ is the classes of equivalence of asymptotic geodesics. For each $z \in \widetilde{M}(\infty)$ and $p$ in $\widetilde{M}$, there is a unique horosphere $Q$, which contains $p$ and is centered at $z$ (see [7] pages 34 and 35 for definitions and details). It is well known that each horosphere in $H^{n}(c)$ is isometric to $\mathbb{R}^{n-1}$.

By using the statements and remarks on pages 201 and 202 of [6], we have the following fact:

Fact 2.6. Consider the 1-parameter family $Q_{t}, t \in \mathbb{R}$, of horospheres of $H^{n}(c)$, centered at $z$ and let $G$ be a connected and closed Lie subgroup of isometries of $H^{n}(c)$ such that $G\left(Q_{0}\right)=Q_{0}$. Then

(a) For each $t$ we have $G\left(Q_{t}\right)=Q_{t}$ and for each orbit $\widetilde{D_{0}}$ in $Q_{0}$, there is an orbit $\widetilde{D}$ in $Q_{t}$ such that $\operatorname{dim} \widetilde{D}=\operatorname{dim} \widetilde{D_{0}}$.

(b) If $V_{0}$ is a totally geodesic orbit in $Q_{0}$, then for each $t \in \mathbb{R}$, there is a totally geodesic orbit $V_{t}$ in $Q_{t}$ such that the following set is a totally geodesic submanifold of $H^{n}$ :

$$
T_{V}=\bigcup_{t} V_{t}
$$

and we have $\operatorname{dim}\left(V_{t}\right)=\operatorname{dim} V_{0}$ (i.e., $\left.\operatorname{dim}\left(T_{V}\right)=\operatorname{dim} V_{t}+1\right)$.

Theorem 2.7 ([6]). Let $G$ be a connected Lie subgroup of the isometries of hyperbolic space $H^{n}$. Then, one of the following assertions is true:

(i) $G$ has a fixed point.

(ii) $G$ has a unique nontrivial totally geodesic orbit.

(iii) All orbits are included in horospheres centered at the same point at infinity.

\section{Results}

Lemma 3.1. Let $M^{2}(c)$ be a complete and connected Riemannian manifold of dimension two and constant negative curvature $c$, which is of cohomogeneityone under the action of a closed and connected subgroup $G$ of isometries. Then, one of the following is true.

(a) $M^{2}(c)$ is simply connected (i.e., $M^{2}(c)=H^{2}(c)$ ). $S^{1}$.

(b) $\pi_{1}\left(M^{2}(c)\right)=\mathbb{Z}$ and there is at least one orbit, which is diffeomorphic to

Proof. We give a proof by using the ideas of [14]. Consider $\widetilde{M}\left(=H^{2}(c)\right)$ and $G^{\prime}$ as in Fact 2.1. $G^{\prime}$ acts by cohomogeneity one on $H^{2}(c)$. If $G^{\prime}$ has a fixed point in $H^{2}(c)$, then it is in fact a singular orbit. But by a theorem in [14], 
there is at most one singular orbit, so the fixed point of $G^{\prime}$ on $H^{2}(c)$ is unique. Thus, by Fact 2.1(d), $M$ is simply connected. Now, suppose that the action of $G^{\prime}$ on $H^{2}(c)$ is without fixed point (all orbits are one dimensional). If $M$ is simply connected we have done. If not, then there is a non-identity element $\delta$ in $\Delta$. Consider the function $f_{\delta}(x)=d^{2}(x, \delta x)$ on $\widetilde{M}$ and let $\gamma$ be a normal geodesic in $\widetilde{M}$. The function $F: \mathbb{R} \rightarrow \mathbb{R}, F(t)=f_{\delta} o \gamma(t)$, is strictly convex (see [14] for definition of normal geodesic and details about convex functions). Thus we have one of the cases below:

Case 1: $F$ has a unique minimum point.

Case 2: $F$ does not have any minimum point.

Case 1: Let $t_{o}$ be the minimum point of $F$ and let $D_{0}=G^{\prime}\left(\gamma\left(t_{0}\right)\right)$. Since $\delta$ commutes with the elements of $G^{\prime}, f_{\delta}$ is constant along orbits. Thus $D_{0}$ is the minimum point set of $f_{\delta}$, so it is totally geodesic (i.e., $D_{0}$ is the image of a geodesic and it is diffeomorphic to $\mathbb{R}$ ). By Theorem 2.7, there is at most one totally geodesic orbit. Thus $D_{0}$ is the unique totally geodesic orbit. Each element of $\Delta$ maps orbits of $\widetilde{M}$ on to orbits. From uniqueness of the totally geodesic orbit $D_{0}$, we get that $\Delta\left(D_{0}\right)=D_{0}$. Now, by Fact 2.3 , we have $\pi_{1}(M)=\mathbb{Z}$, and the orbit $B=\frac{D_{0}}{\Delta}$ in $M$, is diffeomorphic to $\frac{\mathbb{R}}{\mathbb{Z}}=S^{1}$.

Case 2: In this case, for each orbit $D$ in $\widetilde{M}$, we have $\Delta(D)=D$. This is so because, if there are different orbits $D_{1}=G^{\prime}\left(\gamma\left(t_{1}\right)\right)$ and $D_{2}=G^{\prime}\left(\gamma\left(t_{2}\right)\right)$, such that for some $\delta \in \Delta, \delta\left(D_{1}\right)=D_{2}$, then for some $x \in D_{2}$ we would have:

$$
\delta\left(\gamma\left(t_{1}\right)\right)=x \in D_{2},
$$

$f_{\delta}$ is constant along orbits, so $f_{\delta}(x)=f_{\delta}\left(\gamma\left(t_{2}\right)\right)$ and we have

$$
\begin{aligned}
F\left(t_{1}\right) & =d^{2}\left(\gamma\left(t_{1}\right), \delta \gamma\left(t_{1}\right)\right)=d^{2}\left(\delta \gamma\left(t_{1}\right), \delta^{2} \gamma\left(t_{1}\right)\right) \\
& =d^{2}(x, \delta(x))=f_{\delta}(x)=f_{\delta}\left(\gamma\left(t_{2}\right)\right)=F\left(t_{2}\right) .
\end{aligned}
$$

Therefore, $F$ has a minimum point between $t_{1}$ and $t_{2}$. Which is a contradiction. Now consider an orbit $D$ in $\widetilde{M}$. $D$ is one dimensional and simply connected, so it is diffeomorphic to $\mathbb{R}$. $\Delta$ is a discrete group of isometries of $D$, so $\Delta=\mathbb{Z}$. The orbit $\frac{D}{\Delta}$ in $M$ is thus diffeomorphic to $S^{1}$.

Lemma 3.2. Let $M^{n}, n \geq 3$, be a negatively curved and non-simply connected complete Riemannian manifold, which is of cohomogeneity one under the action of a closed and connected Lie group $G$ of isometries. If all orbits of this action is of dimension $n-1$, then there is a positive integer $k$ such that the orbits are diffeomorphic to $\mathbb{R}^{n-1-k} \times T^{k}$ and $M$ is diffeomorphic to $\mathbb{R}^{n-k} \times T^{k}$.

Proof. $M$ does not have any singular orbit (as with Theorem 3.5 of [14], if there is a singular orbit, it must be one dimensional). Thus by Theorem 3.7(a) in [14], each orbit is diffeomorphic to $\mathbb{R}^{n-1-k} \times T^{k}$ and $\frac{M}{G}$ is diffeomorphic to $\mathbb{R}$. Therefore $M$ is diffeomorphic to $\mathbb{R}^{n-k} \times T^{k}$. 
Lemma 3.3. Let $G$ be a connected and closed Lie subgroup of isometries of $H^{n}(c)$ and let $F=\left\{x \in H^{n}(c): G(x)=x\right\}$. If $\operatorname{dim} F \geq 2$, then the cohomogeneity of the action of $G$ on $H^{n}(c)$ is $\geq 3$.

Proof. This lemma is correct in more general case for Riemannian manifolds. But we give a simple proof for our especial case. $F$ is a totally geodesic submanifold of $H^{n}(c)$. Thus, we have $F=W \cap H^{n}(c)$, where $W$ is a time like vector subspace of $\mathbb{R}^{n, 1}$ generated by $\mathrm{F}$. If $x_{1} \in W$, we can easily show that $G\left(x_{1}\right)=x_{1}$. Since $\operatorname{dim} F \geq 2$, then $\operatorname{dim} W \geq 3$. Let $W^{\perp}$ be the orthogonal complement of $W$ in $\mathbb{R}^{n, 1}$. Without loss of generality, we suppose that

$$
W=\mathbb{R}^{m, 1}, W^{\perp}=\mathbb{R}^{n-m}, m \geq 2,
$$

$G$ acts trivially on $W$ so it can be identified with a subgroup of $O(n-m)$. Let

$$
x=x_{1}+x_{2}, x_{1} \in \mathbb{R}^{m, 1}, x_{2} \in \mathbb{R}^{n-m} .
$$

For each $g \in G$, we have

$$
g\left(x_{1}+x_{2}\right)=g\left(x_{1}\right)+g\left(x_{2}\right)=x_{1}+g\left(x_{2}\right) \in x_{1}+\mathbb{R}^{n-m} .
$$

Therefore,

$$
\operatorname{dim} G(x)=\operatorname{dim}\left\{g\left(x_{1}+x_{2}\right): g \in G\right\}=\operatorname{dim}\left\{g x_{2}: g \in G\right\}=\operatorname{dim} G\left(x_{2}\right),
$$

$G$ fixes the origin of $\mathbb{R}^{n-m}$ so for each $x_{2} \in \mathbb{R}^{n-m}$, the orbit $G\left(x_{2}\right)$ is contained in the sphere $S^{n-m-1}(r) \subset \mathbb{R}^{n-m}, r=\left|x_{2}\right|$. Thus we have

$$
\operatorname{dim} G\left(x_{2}\right) \leq n-m-1 \Rightarrow \operatorname{dim} G(x) \leq n-m-1 .
$$

Therefore the cohomogeneity of the action of $G$ on $H^{n}(c)$ is $\geq m+1 \geq 3$.

Lemma 3.4. Let $G$ be a connected and closed Lie subgroup of isometries of $H^{n}(c)$ and suppose that there exists a 1-parameter family of horospheres $Q_{t}$ centered at a point $z$, such that the action of $G$ on $Q_{0}$ is of cohomogeneity one. Then, for each $t$ we have $G\left(Q_{t}\right)=Q_{t}$ and one of the following is true:

(1) For each $t \in \mathbb{R}$, the orbits of $Q_{t}$ are isometric to $\mathbb{R}^{n-2}$.

(2) There exists an integer $m, 0<m<n-2$, such that for each $t \in \mathbb{R}, Q_{t}$ has one orbit isometric to $\mathbb{R}^{m}$ and the other orbits of $Q_{t}$ are diffeomorphic to $S^{n-2-m} \times \mathbb{R}^{m}$.

Proof. Since the cohomgeneity of the action of $G$ on $Q_{0}$ is one, then by Fact 2.6(a) for each $t \in \mathbb{R}$, the action of $G$ on $Q_{t}$ is of cohomogeneity one. $Q_{0}$ is isometric to $\mathbb{R}^{n-1}$ so by Theorem 2.2 we have the two cases below:

(1) Each orbit of $Q_{0}$ is isometric to $\mathbb{R}^{n-2}$.

(2) One orbit of $Q_{0}$ is isometric to $\mathbb{R}^{m}, 0<m<n-2$, and the other orbits are diffeomorphic to $S^{n-2-m} \times \mathbb{R}^{m}$.

By Fact 2.6(a) (and by dimensional reasons), it is easy to show that (1) or (2), which is true for $Q_{0}$, must be true for all $Q_{t}, t \in \mathbb{R}$. 
Lemma 3.5. Let $M^{n}(c)$ be a Riemannian G-manifold of constant negative curvature $c, \pi: H^{n}(c) \rightarrow M^{n}(c)$ be the covering map, and $G^{\prime}$ be the covering group of $G$ as in Fact 2.1. Also let $\tilde{N}$ be a totally geodesic submanifold of $H^{n}(c)$ such that $\operatorname{dim} \tilde{N}=m+1, m \geq 1, G^{\prime}(\tilde{N})=\tilde{N}, \Delta(\tilde{N})=\tilde{N}, N=k(\tilde{N})$, all $G^{\prime}-$ orbits of $\tilde{N}$ be of dimension $m$. Then, $N$ is a totally geodesic cohomogeneity-one $G$-submanifold of $M$ and one of the following assertions is true:

(I) $M$ is simply connected.

(II) $\pi_{1}(M)=\mathbb{Z}$ and there is an orbit $B$ in $M$ which is diffeomorphic to $S^{1}$.

(III) $\pi_{1}(M)=\mathbb{Z}^{k}$ for some positive integer $k$. Each orbit in $N$ is diffeomorphic to $\mathbb{R}^{m-k} \times T^{k}$ and $N$ is diffeomorphic to $\mathbb{R}^{m-k+1} \times T^{k}$.

Proof. $\tilde{N}$ is isometric to $H^{m+1}(c)$. If $m=1$, then by Lemma 3.1, one of the following is true:

(I) $N$ is simply connected.

(II) $\pi_{1}(N)=\mathbb{Z}$ and there is an orbit $B \subset N$ which is diffeomorphic to $S^{1}$.

If $m \geq 2$, then by Lemma 3.2 we get that

(III) Each orbit in $N$ is diffeomorphic to $T^{k} \times \mathbb{R}^{m-k}$ for some $k$, and $N$ is diffeomorphic to $T^{k} \times \mathbb{R}^{m+1-k}$ (i.e., $\pi_{1}(N)=\mathbb{Z}^{k}$ ).

Since $\Delta(\tilde{N})=\tilde{N}$, then $\pi_{1}(M)=\pi_{1}(N)$, and we get the results.

Theorem 3.6. Let $M^{n}(c), n \geq 3$, be a complete and connected Riemannian manifold of constant negative sectional curvature $c$, and let $G$ be a connected and closed Lie subgroup of isometries, which acts by cohomogeneity two on $M$. Then, one of the following is true:

(a) $M$ is simply connected, i.e., $M=H^{n}(c)$.

(b) Each orbit is diffeomorphic to $\mathbb{R}^{m} \times T^{n-2-m}$ for some nonnegative integer $m$, and $M$ is a union of totally geodesic cohomogeneity-one Riemannian $G$ submanifolds.

(c) $\pi_{1}(M)=\mathbb{Z}$ and there exists a set $B$ in $M$, which is diffeomorphic to $S^{1}$ and $B$ is either an orbit or $B=F i x(G, M)$.

(d) $\pi_{1}(M)=\mathbb{Z}^{k}$ for some positive integer $k$ and $M$ is a union of the following two types of orbits:

(d1) The orbits which are diffeomorphic to $\mathbb{R}^{m-k} \times T^{k}$ for some positive integer $m$. A union of this type of orbits is a totally geodesic submanifold of $M$.

(d2) The orbits covered by $S^{n-2-m} \times \mathbb{R}^{m}$.

Proof. As in Fact 2.1, let $G^{\prime}$ be the connected covering group of $G$ which acts by cohomogeneity two on $H^{n}(c)$, the universal Riemannian covering manifold of $M$. We denote the covering maps by

$$
\pi: H^{n}(c) \rightarrow M^{n}(c), \pi^{\prime}: G^{\prime} \rightarrow . G
$$

By Theorem 2.7, we have the three cases below:

(i) $G^{\prime}$ has a fixed point.

(ii) $G^{\prime}$ has a unique nontrivial totally geodesic orbit. 
(iii) All orbits are included in horospheres centered at the same point at infinity.

We study each case separately.

(i): Let $F=\left\{x \in H^{n}(c): G^{\prime}(x)=x\right\}$. If $\operatorname{dim} F \geq 2$, then by Lemma 3.3, the cohomogeneity of the action of $G^{\prime}$ on $H^{n}(c)$ is $\geq 3$, which is a contradiction. If $\operatorname{dim}(F)=1$, then, from the fact that fixed point set is a totally geodesic submanifold, we get that $F$ is the image of a geodesic $\lambda$. If $M$ is simply connected, then we have part (a) of the theorem. If $M$ is not simply connected, recall that each $\delta$ in $\Delta$ commutes with the elements of $G^{\prime}$. Thus $\Delta$ maps fixed points of $G^{\prime}$ on to fixed points, and we have $\Delta(\lambda)=\lambda$. Now by Fact 2.3 we get that $\pi_{1}(M)=\mathbb{Z}$, and by Fact $2.1\left(\mathrm{c}\right.$ ), the set $B=\frac{F}{\Delta}$ (which is diffeomorphic to $\left.\frac{\mathbb{R}}{\mathbb{Z}}=S^{1}\right)$ is equal to $\operatorname{Fix}(G, M)$. This is the part (c) of the theorem.

Now let $\operatorname{dim}(F)=0$. Since $F$ is connected, it has only one point. Then, by Fact $2.1(\mathrm{~d})$, we get that $M=H^{n}(c)$. This is the part (a) of the theorem.

(ii): Let $P$ be the unique totally geodesic orbit of the action of $G^{\prime}$ on $H^{n}(c)$. If $P=H^{n}(c)$, then the action is not of cohomogeneity two. Thus we have $\operatorname{dim} P<n$. Each $\delta$ in $\Delta$ maps $G^{\prime}$-orbits of $H^{n}(c)$ onto $G^{\prime}$-orbits. Since $P$ is the unique totally geodesic orbit, we have $\Delta(P)=P$. If $\operatorname{dim} P=1$, then $P$ is a geodesic in $H^{n}(c)$. Using Fact 2.3, we get that $\Delta=\mathbb{Z}$ and the orbit $B=\pi(P)=\frac{P}{\mathbb{Z}}$ is diffeomorphic to $\frac{\mathbb{R}}{\mathbb{Z}}=S^{1}$. These yield to the part (c) of the theorem. Now, let $\operatorname{dim} P>1$ and consider the orbit $\pi(P)$ in $M$. Since $P$ is a totally geodesic $G^{\prime}$-orbit in $H^{n}(c), \pi(P)$ is a totally geodesic $G$-orbit in $M$. Thus $\pi(P)$ is homogeneous and of negative curvature, and by Theorem 2.4 , it is simply connected. Therefore, the covering map $\pi: P \rightarrow \pi(P)$ is trivial. Since $\Delta$ is the deck transformation group of coverings $P \rightarrow \pi(P)$ and $H^{n}(c) \rightarrow M$, $\Delta$ is trivial and $M$ is simply connected (part (a) of the theorem).

(iii): Let $Q_{t}$ be a 1-parameter family of horospheres, such that $G^{\prime}\left(Q_{t}\right)=$ $Q(t)$. Since the action of $G^{\prime}$ on $H^{n}(c)$ is of cohomogeneity two, then at least for some $t$, say $t=0$, the action of $G^{\prime}$ on $Q_{0}$ is of cohomogeneity one. Thus, by Lemma 3.4, one of the following is true.

(iii1) Each orbit in $Q_{t}, t \in \mathbb{R}$, is isometric to $\mathbb{R}^{n-2}$.

(iii2) There exists an integer $m, 0<m<n-2$, such that one orbit of $Q_{t}, t \in \mathbb{R}$, is isometric to $\mathbb{R}^{m}$ and the other orbits of $Q_{t}$ are diffeomorphic to $S^{n-2-m} \times \mathbb{R}^{m}$.

We study (iii) and (iii2) separately.

(iii1) Consider an orbit $D$ in $M$. We have $D=\pi(V)$. Where $V$ is a $G^{\prime}$-orbit in $H^{n}(c)$. Since $V$ is isometric to $\mathbb{R}^{n-2}, D$ is flat (and homogeneous). Thus, by Theorem $2.5, D$ is diffeomorphic to $\mathbb{R}^{m} \times T^{n-2-m}$ for some $m \geq 0$.

Now, consider an orbit $V_{0}$ in $Q_{0}$. Since $V_{0}\left(=\mathbb{R}^{n-2}\right)$ is totally geodesic in $Q_{0}\left(=\mathbb{R}^{n-1}\right)$, then, by using the Fact 2.6, we get that for each $t$, there is a $G^{\prime}-$ orbit $V_{t}$ in $Q_{t}$, such that $T_{V_{0}}=\bigcup_{t} V_{t}$ is a totally geodesic cohomogeneity-one $G^{\prime}$-submanifold of $H^{n}(c)$. Therefore $\pi\left(T_{V_{0}}\right)$ is a totally geodesic cohomogeneityone $G$-submanifold of $M$. Since $H^{n}(c)=\bigcup_{V_{0}} T_{V_{0}}$ (where the union is on all 
orbits $V_{0}$ in $\left.Q_{0}\right)$, then $M=\bigcup_{V_{0}} \pi\left(T_{V_{0}}\right)$. This means that $M$ is a union of totally geodesic cohomogeneity-one Riemannian $G$-submanifolds. These yield to the part (b) of the theorem.

(iii2) Let $V_{0}$ be the unique orbit of $Q_{0}$ which is isometric to $\mathbb{R}^{m}, 0<$ $m<n-2$. Consider the orbit $V_{t}$ in $Q_{t}, t \in \mathbb{R}$, as in Fact 2.6(b). Since $\operatorname{dim} V_{t}=\operatorname{dim} V_{0}=m$, then $V_{t}$ is the unique orbit in $Q_{t}$, which is isometric to $\mathbb{R}^{m}$. By Fact 2.6(b), the set $\widetilde{N}=\bigcup_{t} V_{t}$ is a totally geodesic $G^{\prime}$-submanifold of $H^{n}(c)$. So the set $N=\pi(\tilde{N})$ is a totally geodesic $G$-submanifold of $M$. We have

$$
H^{n}(c)=\tilde{N} \bigcup\left(H^{n}(c)-\tilde{N}\right),
$$

$G^{\prime}$-orbits of $\widetilde{N}$ are isometric to $\mathbb{R}^{m}$ and the orbits in $\left(H^{n}(c)-\widetilde{N}\right)$ are diffeomorphic to $\mathbb{R}^{m} \times S^{n-2-m}$. Since each $\delta$ in $\Delta$ maps orbits to orbits, by dimensional reasons we have

$$
\Delta(\widetilde{N})=\widetilde{N}, \Delta\left(H^{n}(c)-\tilde{N}\right)=H^{n}(c)-\tilde{N} .
$$

We can, therefore, get one of the assertions (I), (II) or (III) of Lemma 3.5. (I) and (II) yield to parts (a) and (c) of the theorem. If (III) is true, then we have $\pi_{1}(M)=\mathbb{Z}^{k}$, and the orbits of $N$ are diffeomorphic to $T^{k} \times \mathbb{R}^{n-k}$. We have also

$$
M=\frac{H^{n}(c)}{\Delta}=\frac{\tilde{N}}{\Delta} \bigcup \frac{H^{n}(c)-\tilde{N}}{\Delta}=N \bigcup \frac{H^{n}(c)-\tilde{N}}{\Delta} .
$$

The orbits in $\frac{H^{n}(c)-\widetilde{N}}{\Delta}$ are covered by $\mathbb{R}^{m} \times S^{n-2-m}$. Thus we get the part (d) of the theorem.

It is now easy to get the following corollary, from the proof of Theorem 3.6.

Corollary 3.7. If $H^{n}(c)$ is of cohomogeneity two under the action of a closed and connected Lie group $G^{\prime}$ of isometries, then one of the following assertions is true:

(a1) $G^{\prime}$ has fixed point.

(a2) There is a unique totally geodesic orbit.

(a3) All orbits are isometric to $\mathbb{R}^{n-2}$.

(a4) There exists a positive integer $m$, such that each orbit is either isometric to $\mathbb{R}^{m}$ or diffeomorphic to $S^{n-m-2} \times \mathbb{R}^{m}$.

\section{References}

[1] A. V. Alekseevsky and D. V. Alekseevsky, G-manifolds with one-dimensional orbit space, Lie groups, their discrete subgroups, and invariant theory, 1-31, Adv. Soviet Math., 8, Amer. Math. Soc., Providence, RI, 1992.

[2] _ Riemannian G-manifold with one-dimensional orbit space, Ann. Global Anal. Geom. 11 (1993), no. 3, 197-211.

[3] G. E. Bredon, Introduction to Compact Transformation Groups, Academic Press, New York-London, 1972. 
[4] J. Berndt, S. Console, and C. Olmos, Submanifolds and Holonomy, Chapman \& Hall/CRC Research Notes in Mathematics, 434. Chapman \& Hall/CRC, Boca Raton, FL, 2003.

[5] M. P. do Carmo, Riemannian Geometry, Birkhäuser Boston, Inc., Boston, MA, 1992.

[6] A. J. Di Scala and C. Olmos, The geometry of homogeneous submanifolds of hyperbolic space, Math. Z. 237 (2001), no. 1, 199-209.

[7] P. Eberlein, Geodesic follows in manifolds of nonpositive curvature, http://www.math. unc.edu/faculty/pbe/ams.

[8] S. Kobayashi, Homogeneous Riemannian manifolds of negative curvature, Tohoku Math. J. (2) 14 (1962), 413-415.

[9] S. Kobayashi and K. Nomizu, Foundations of Differential Geometry. Vol I, Vol II, Interscience Publishers John Wiley \& Sons, Inc., New York, 1963, 1969.

[10] P. W. Michor, Isomrtric actions of Lie groups and invariants, Lecture course at the university of Vienna, 1996/1997, http://www.mat.univie.ac.at/ michor/tgbook.ps.

[11] R. Mirzaie, Cohomogeneity two actions on flat Riemannian manifolds, Acta Math. Sin. (Engl. Ser.) 23 (2007), no. 9, 1587-1592.

[12] R. Mirzaie and S. M. B. Kashani, On cohomogeneity one flat Riemannian manifolds, Glasg. Math. J. 44 (2002), no. 2, 185-190.

[13] B. O'Neil, Semi-Riemannian Geometry: With Applications to Relativity, Pure and Applied Mathematics, 103. Academic Press, Inc. [Harcourt Brace Jovanovich, Publishers], New York, 1983.

[14] E. Podestá and A. Spiro, Some topological properties of cohomogeneity one manifolds with negative curvature, Ann. Global Anal. Geom. 14 (1996), no. 1, 69-79.

[15] C. Searle, Cohomogeneity and positive curvature in low dimensions, Math. Z. 214 (1993), no. 3, 491-498.

[16] J. A. Wolf, Spaces of Constant Curvature, McGraw-Hill, New York, 1967.

[17] Homogeneity and bounded isometries in manifolds of negative curvature, Illinois J. Math. 8 (1964), 14-18.

Department of Mathematics

FACULTY OF SCIENCES

I. KH. INTERNATIONAL UNIVERSity

QAZVIN, IRAN

E-mail address: r_mirzaioe@yahoo.com 